\title{
稻田 $\mathrm{CH}_{4}$ 传输的碳同位素分馏研究
}

\author{
张广斌 ${ }^{(1)}$, 纪洋 ${ }^{(1)}$, 刘刚 ${ }^{(1)}$ ，马静 ${ }^{(1)}$, 徐华 ${ }^{(1)}$ \\ (1) 中国科学院南京土壤研究所土壤与农业可持续发展国家重点实验室, 南京 210008; \\ (2) 中国科学院大学, 北京 100049 \\ * 联系人, E-mail: hxu@issas.ac.cn
}

收稿日期: 2013-07-29; 接受日期: 2013-12-29; 网络版发表日期: 2014-07-24

中国科学院战略性先导科技专项(编号: XDA05020200)、国家自然科学基金项目(批准号：41071169)、国家国际科技合作专项项目(编号: 2012DFG90290)和公益性行业(农业)科研专项(编号: 201103039)资助

\begin{abstract}
摘要 为进一步推动稳定性碳同位素自然丰度法在我国稻田 $\mathrm{CH}_{4}$ 研究中的应用, 通过 田间试验研究了水稻生长期和非水稻生长期 $\mathrm{CH}_{4}$ 传输的碳同位素分馏, 重点介绍了两种 新的观测稻田 $\mathrm{CH}_{4}$ 传输碳同位素分馏系数 $\varepsilon$ 传啝方法. 结果表明: 密闭箱+注射器法能较 好地测定非水稻生长期稻田 $\varepsilon$ 传轵, 此时 $\varepsilon$ 传较为田间排放的 $\delta^{13} \mathrm{CH}_{4}$ 值减去表层水中的 $\delta^{13} \mathrm{CH}_{4}$ 值(-6.7\% o - 3\%o). 水稻生长期, $\varepsilon$ 传输可通过三种方法获得, 分别是田间排放的 $\delta^{13} \mathrm{CH}_{4}$ 值减

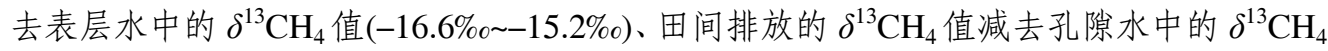

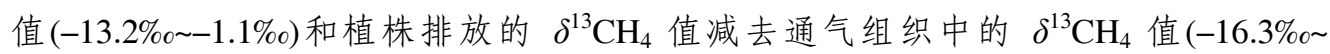
$-10.9 \%$ ) , 但前两种方法均存在较大的不确定性, 只有最后一种方法一一分隔+切割法, 既科学, 又可靠, 且能获得准确的观测结果.
\end{abstract}

关键词

稻田

$\mathrm{CH}_{4}$ 传输

同位素分馏系数

密闭箱+注射器法

分隔+切割法
$\mathrm{CH}_{4}$ 是大气中重要的温室气体, 2010 年大气中 $\mathrm{CH}_{4}$ 浓度达到 $1808 \mathrm{ppb}$ (世界气象组织, 2011, http:// www.wmo.int/pages/mediacentre/press_releases/documents/ GHGbulletin.pdf; $\left.1 \mathrm{ppb}=1 \mu \mathrm{g} \mathrm{L}{ }^{-1}\right)$. 稻田是大气 $\mathrm{CH}_{4}$ 的重要排放源, 稻田 $\mathrm{CH}_{4}$ 排放是土壤中 $\mathrm{CH}_{4}$ 产生、氧 化和传输的净效应. $\mathrm{CH}_{4}$ 产生是 $\mathrm{CH}_{4}$ 排放的先决条件, $\mathrm{CH}_{4}$ 氧化对减少 $\mathrm{CH}_{4}$ 排放具有重要意义, $\mathrm{CH}_{4}$ 传输是 土壤中 $\mathrm{CH}_{4}$ 进入大气的重要过程. 由于土壤中 $\mathrm{CH}_{4}$ 的产生、氧化和传输几乎同时发生, 很长一段时间内 土壤科学工作者难以很好地单独研究上述各过程. 近十几年来, 稳定性碳同位素自然丰度法被广泛运 用到稻田 $\mathrm{CH}_{4}$ 排放研究中, 尤其是与稻田 $\mathrm{CH}_{4}$ 排放密 切相关的 $\mathrm{CH}_{4}$ 产生、氧化和传输各过程(Chanton 等,
1997; Tyler 等, 1997; Bilek 等, 1999; Krüger 等, 2002; Conrad 和 Klose, 2005). 稻田土壤产生的 $\mathrm{CH}_{4}$ 通过植 物通气组织、气泡和液相扩散等途径向大气排放, 而 $\mathrm{CH}_{4}$ 从土壤中向大气释放时会发生碳同位素分馏, 即 ${ }^{12} \mathrm{CH}_{4}$ 较 ${ }^{13} \mathrm{CH}_{4}$ 能更快地被传输而释放到大气中. $\mathrm{CH}_{4}$ 传输的碳同位素分馏系数(通常用 $\varepsilon$ 传输表示, 为传输 过程所导致的 $\delta^{13} \mathrm{CH}_{4}$ 变化)是稳定性碳同位素自然丰 度法研究 $\mathrm{CH}_{4}$ 氧化率不可或缺的关键因子(张广斌等, 2009), 因此, 获得准确、可靠的 $\varepsilon$ 传制是应用该方法研 究稻田 $\mathrm{CH}_{4}$ 排放的重要前提.

大量研究发现, 水稻植株向大气传输 $\mathrm{CH}_{4}$ 时会 发生较强烈的碳同位素分馏, 且产生的同位素分馏 系数 $\varepsilon$ 传输一般为 $-18 \%$ - $9.0 \%$ (Chanton 等, 1997; Tyler

中文引用格式: 张广斌, 纪洋, 刘刚, 等. 2014. 稻田 $\mathrm{CH}_{4}$ 传输的碳同位素分馏研究. 中国科学: 地球科学, 44: 2016-2021

英文引用格式: Zhang G B, Ji Y, Liu G, et al. 2014. Carbon isotope fractionation during $\mathrm{CH}_{4}$ transport in paddy fields. Science China: Earth Sciences, 57: 16641670, doi: 10.1007/s11430-014-4879-3 
等, 1997; Bilek 等, 1999; Krüger 等, 2002; Conrad 和 Klose, 2005). 通常情况下, 水稻植株的 $\varepsilon$ 传输可由田间 排放的 $\delta^{13} \mathrm{CH}_{4}$ 值减去土壤孔隙水中的 $\delta^{13} \mathrm{CH}_{4}$ 值或由 田间排放的 $\delta^{13} \mathrm{CH}_{4}$ 值减去水稻植株通气组织中的 $\delta^{13} \mathrm{CH}_{4}$ 值获得(Chanton 等, 1997; Tyler 等, 1997; Bilek 等, 1999; Krüger 等, 2002; Conrad 和 Klose, 2005), 但 以往研究方法存在一些缺陷, 可能影响计算结果的 准确度. 孔隙水中的 $\mathrm{CH}_{4}$ 可能包含未被氧化的 $\mathrm{CH}_{4}$, 因此其 $\delta^{13} \mathrm{CH}_{4}$ 并不能很好地代表已被甲烷氧化细菌 氧化但还未经水稻植株传输的 $\delta^{13} \mathrm{CH}_{4}$; 通常用针直 接取几棵水稻植株通气组织里的气样混合后分析其 $\delta^{13} \mathrm{CH}_{4}$ 值(Chanton 等, 1997; Tyler 等, 1997; Bilek 等, 1999; Krüger 等, 2002), 这种方式取得的 $\mathrm{CH}_{4}$ 可能包 含植株上部已经扩散分馏的 $\mathrm{CH}_{4}$, 多次采样还可能混 入空气带来误差. 如尝试在尽可能低的水层顶部用 隔板将水稻植株与其下方的土壤及水层完全分隔开 来, 隔板上的孔隙允许稻茎完整无损地从中穿过, 同 时密封不让下方的气体从孔隙中扩散至上层空间, 然后将水稻植株隔板以上的部分全部切除, 用密闭 箱法观测刚进入水稻通气组织里的 $\delta^{13} \mathrm{CH}_{4}$. 这种分 隔+切割观测方法应能避免植株上部或空气的影响, 获得更准确的 $\varepsilon$ 传制值, 但需要严格设计的田间试验予 以验证. 目前有关稻田 $\mathrm{CH}_{4}$ 传输碳同位素分馏的报 道均来自于西方国家稻田, 而关于我国稻田 $\mathrm{CH}_{4}$ 传 输碳同位素分馏的研究还是空白, 这严重地阻碍了 采用稳定性碳同位素自然丰度法研究我国稻田 $\mathrm{CH}_{4}$ 的排放过程, 特别是稻田 $\mathrm{CH}_{4}$ 氧化率的测定.

$\mathrm{CH}_{4}$ 通过气泡和液相扩散途径向大气传输的过 程中也会发生碳同位素分馏(Chanton, 2005). Happell 等(1994)在佛罗里达州森林沼泽的研究结果表明, $\mathrm{CH}_{4}$ 通过扩散传输经过气-水界面时发生 $2 \%$ 3\%o的 碳同位素分馏. Knox 等(1992)和 Happell等(1995)通过 室内研究发现, $\mathrm{CH}_{4}$ 从静止的纯净水中排放会产生 $1 \%$ 的碳同位素分馏. 迄今为止, 有关稻田类似的研 究尚属空白. 实施全年持续淹水处理的稻田非水稻 生长期, 土壤中的 $\mathrm{CH}_{4}$ 通过气泡和液相扩散传输释 放至大气中, 其传输途径与水稻生长期存在较大差 异, 因此其 $\varepsilon$ 传输应与水稻生长期的不同, 且观测方法 也应该存在一定差异. 以往研究仅报道了水稻生长

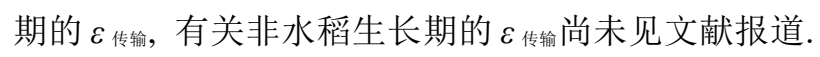
采用何种方法测定非水稻生长期的 $\varepsilon$ 传赫是本试验又 一项重要的研究内容. 非水稻生长期, $\mathrm{CH}_{4}$ 从土壤中
向大气释放的过程中会在土水界面会发生强烈的 $\mathrm{CH}_{4}$ 氧化作用, 故而表层水中的 $\delta^{13} \mathrm{CH}_{4}$ 能在很大程度 上代表已被甲烷氧化细菌氧化但还未经气泡或液相 扩散传输的 $\delta^{13} \mathrm{CH}_{4}$. 因此, 通过同时观测田间排放的 $\delta^{13} \mathrm{CH}_{4}$ (静态密闭箱法采集气样) 和表层水中的 $\delta^{13} \mathrm{CH}_{4}$ (注射器采集水样, 并最终获得水中气样), 就 可以评估经气泡和液相扩散传输 $\mathrm{CH}_{4}$ 时发生的碳同 位素分馏. 这种密闭箱+注射器观测方法有望获得准 确、可靠的 $\varepsilon$ 传输, 但需要田间试验数据予以验证.

为此, 本文通过田间试验研究水稻生长期和非 水稻生长期 $\mathrm{CH}_{4}$ 传输的碳同位素分馏, 并重点介绍 两种新的观测稻田 $\mathrm{CH}_{4}$ 传输碳同位素分馏系数 $\varepsilon$ 传输 的方法, 以填补国内该项研究的空白. 同时通过比较 以往研究方法及其观测结果, 深入探讨这两种方法 的可行性, 以进一步推动稳定性碳同位素自然丰度 法在我国稻田 $\mathrm{CH}_{4}$ 研究中的广泛应用.

\section{1 材料与方法}

\section{1 试验地点}

田间试验在江苏省句容市白兔镇进行 $\left(31^{\circ} 58^{\prime} \mathrm{N}\right.$, $\left.119^{\circ} 18^{\prime} \mathrm{E}\right)$. 该地位于环太湖地区典型稻麦轮作区, 属 北亚热带季风气候. 年平均气温约为 $15^{\circ} \mathrm{C}$, 年平均 降水量约为 $1020 \mathrm{~mm}$. 供试土壤为发育于下蜀黄土 的爽水性水稻土, 土壤 ( 0 15 $\mathrm{cm}$ 土层)有机碳含量为 $9.6 \mathrm{~g} \mathrm{~kg}^{-1}$, 全氮含量为 $1.0 \mathrm{~g} \mathrm{~kg}^{-1}$, 土壤有机碳的 $\delta^{13} \mathrm{C}$ 为 $-27.4 \%$.

\section{2 试验设计}

2008 年 11 月 2 日水稻收割后, 稻田内根茬和杂 草被清理干净，并于 11 月 18 日开始淹水，直到 2009 年 10 月 15 日, 期间保持至少 $2 \mathrm{~cm}$ 深的水层. 水稻(华 粳 3 号, Oryza sativa L) 于 5 月 25 日育种, 6 月 26 日移 栽, 11 月 3 日收割. 稻田施用尿素为 $300 \mathrm{~kg} \mathrm{~N} \mathrm{hm}^{-2}$, 按基肥: 分菜肥: 穗肥为 $2: 1: 1$ 施用, 磷、钾肥分别为 $450 \mathrm{~kg} \mathrm{hm}^{-2}$ 的过磷酸钻和 $225 \mathrm{~kg} \mathrm{~h}^{-2}$ 的氯化钾, 作为 基肥一次性施入. 基肥、分菜肥和穗肥分别于 6 月 26 日、7月 17 日和 8 月 16 日施用. 稻田 10 月 16 号排水 落干直到收获. 试验小区 $(3 \mathrm{~m} \times 4 \mathrm{~m})$ 设置三次重复.

\section{3 样品采集与测定}

田间 $\mathrm{CH}_{4}$ 气样用静态箱采集, 采样时间为上午 
9:00 11:00, 采样时将静态箱罩在事先埋入各试验 小区土壤 $15 \mathrm{~cm}$ 深的 $\mathrm{PVC}$ 底座上. 为测定排放 $\mathrm{CH}_{4}$ 的稳定性碳同位素组成 $\left(\delta^{13} \mathrm{CH}_{4 \text { (排放) }}\right)$, 观测到 $\mathrm{CH}_{4}$ 排 放后每隔 10 15 天采样 1 次. 静态箱密封后, 用小抽 气原将箱内气体导入到事先已抽真空的 $500 \mathrm{~mL}$ 气袋 (外被一层厚铝䈃复合膜, 中国大连德霖气体包装有 限公司提供)中, 共采 2 次样, 静态箱关闭 $3 \sim 5 \mathrm{~min}$ 后 第一次采样, $2 \mathrm{~h}$ 后第二次采样(Zhang 等, 2011). $\delta^{13} \mathrm{CH}_{4 \text { (井放) }}(\%)$ ) 则根据前后两次观测到的 $\mathrm{CH}_{4}$ 浓度及 其对应的稳定性碳同位素组成计算获得:

$$
\delta^{13} \mathrm{CH}_{4 \text { (排放) }}=[(B \times b)-(A \times a)] /(B-A),
$$

式中, $A$ 和 $B$ 分别为第一次和第二次所采气样的 $\mathrm{CH}_{4}$ 浓度 $\left(\mu \mathrm{L} \mathrm{L} \mathrm{L}^{-1}\right), a$ 和 $b$ 分别为对应样品的稳定性碳同位 素组成 $\delta^{13} \mathrm{CH}_{4}(\%)$.

土壤溶液用根际土壤溶液取样器 (Rhizon soil moisture sampler, 简称 Rhizon SMS)采集(Zhang 等, 2011), 间隔 15 30 天采样一次. Rhizon SMS 主要由 多孔聚酯管(长 $10 \mathrm{~cm}$, 外径 $2.5 \mathrm{~mm}$ ) 和 PVC 管(长 100 $\mathrm{cm}$, 外径 $2.7 \mathrm{~mm}$ )两部分组成. 多孔聚酯管于 2008 年 11 月 18 水平埋入各小区土壤 $10 \mathrm{~cm}$ 深处直至试验结 束. 每次取样之前, 先用 $18 \mathrm{~mL}$ 真空瓶抽取约 $5 \mathrm{~mL}$ 的土壤溶液以冲刷洗净取样器, 然后再用另一只 18 $\mathrm{mL}$ 真空瓶采取 $10 \mathrm{~mL}$ 左右样品. 同时用注射器取 10 $\mathrm{mL}$ 土壤表层水, 并迅速注入 $18 \mathrm{~mL}$ 真空瓶. 之后将
样品瓶内充入适量纯 $\mathrm{N}_{2}$, 使之维持在 $1 \mathrm{~atm}$. 震荡、 取气, 备测 $\mathrm{CH}_{4}$ 浓度及其稳定性碳同位素组成.

水稻植株割苗前、后的 $\mathrm{CH}_{4}$ 气样用特制静态箱 采集, 采样装置如图 1 所示. 采样底座为 PVC 材质的 无底圆桶, 高 $30 \mathrm{~cm}$, 直径 $17 \mathrm{~cm}$. 圆桶上端周围为 30 $\mathrm{cm} \times 30 \mathrm{~cm}$ 的正方形 $\mathrm{PVC}$ 板并辅有深 $5 \mathrm{~cm}$ 的水槽, 用 以采样时加水密封采样箱. 采样箱为透明的有机玻 璃箱, 大小为 $30 \mathrm{~cm} \times 30 \mathrm{~cm} \times 100 \mathrm{~cm}$. 采样前 7 10 天, 将底座插入稻田土壤中, 并使得一株水稻恰好在圆 桶的正中央, 同时保证圆桶上端与土壤表层的距离 不超过 $2 \mathrm{~cm}$. 采样时, 用带圆孔(孔径 $4 \mathrm{~cm}$ ) 的 PVC 隔 板 (直径 $18 \mathrm{~cm}$ ) 从圆桶上端将水稻植株分隔成上下两 部分, 然后将(b)箱内水稻植株隔板以上的部分全部 切除, 通过对比观测水稻植株割苗前(a)、后(b)采样箱 内 $\mathrm{CH}_{4}$ 稳定性碳同位素组成的变化来获得 $\mathrm{CH}_{4}$ 传输 的碳同位素分馏系数.

样品 $\mathrm{CH}_{4}$ 浓度用带有氢离子火焰检测器的气相 色谱(岛津 GC-12A) 分析, 样品中 $\mathrm{CH}_{4}$ 的稳定性碳同 位素组成采用带有预浓缩装置 (pre-Con) 的质谱仪 (MAT-253)测定(曹亚澄等, 2008; Zhang 等, 2011), 均 三次重复. pre-Con 是一种全自动预 GC 浓缩装置, 由 样品瓶、化学阱、冷阱、燃烧反应器和六通阀 5 个部 分组成. 待测样品装入样品瓶后, 用 $\mathrm{He}$ 气流将样品 吹入化学阱和冷阱以去除杂质, 仅挥发性组份 $\left(\mathrm{N}_{2}\right.$,

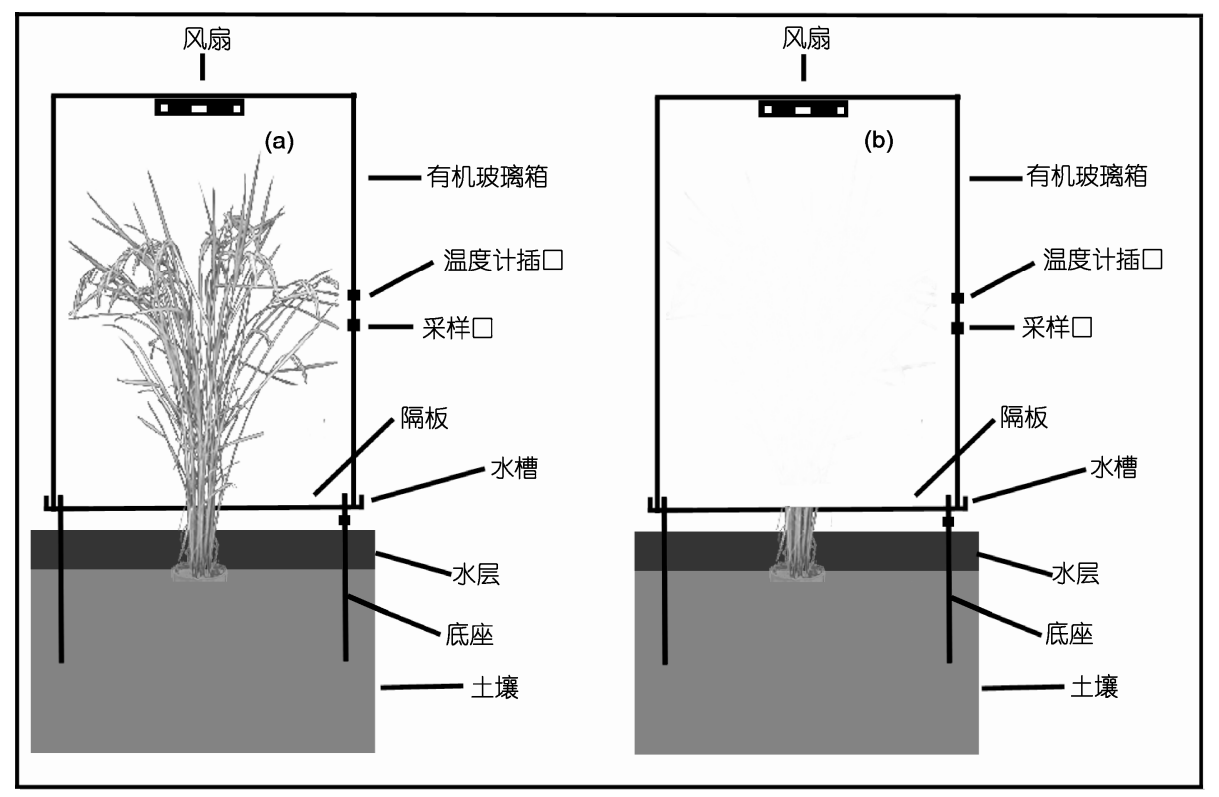

图 1 水稻植株传输 $\mathrm{CH}_{4}$ 的试验装置示意图 
$\mathrm{O}_{2}, \mathrm{Ar}$ 和 $\mathrm{CH}_{4}$ )进入到燃烧反应器中, 在其间样品中 $\mathrm{CH}_{4}$ 被氧化成 $\mathrm{CO}_{2}$ 和 $\mathrm{H}_{2} \mathrm{O}$, 随后流入 $\mathrm{GC}$ 进行进一步 分离. 最终分离出来的 $\mathrm{CO}_{2}$ 进入热电-菲尼根(ThermoFinnigan)MAT-253 同位素质谱仪进行测定. 参比气体 为高纯钢瓶 $\mathrm{CO}_{2}$ 气体, 经用有证标准物 GBW 04407 炭 黑标定, 其 $\delta^{13} \mathrm{C}_{\mathrm{PDB}}$ 值为-23.73\%o(PDB 系南卡罗来纳 州白严系皮狄组美洲拟箭石). 对钢瓶压缩空气中 $\mathrm{CH}_{4}$ 的 $\delta^{13} \mathrm{C}_{\mathrm{PDB}}$ 值连续测量 9 次, 标准差为 $\pm 0.196 \%$.

\section{2 讨论与结果}

非水稻生长期后期，田间排放的 $\delta^{13} \mathrm{CH}_{4}$

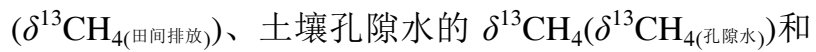

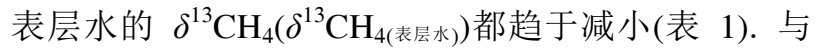

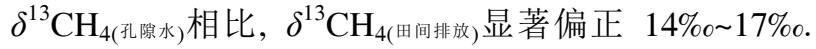

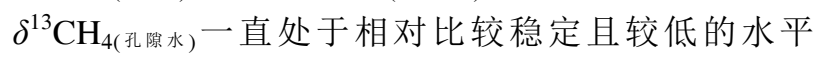
(-65\%o左右)(Zhang 等, 2011), 这说明非水稻生长期 不种植作物使得土壤孔隙水中的 $\mathrm{CH}_{4}$ 受到植株根系

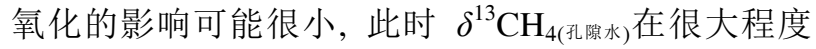
上可代表田间产生的 $\delta^{13} \mathrm{CH}_{4}$. 因此, 非水稻生长期采 用田间排放的 $\delta^{13} \mathrm{CH}_{4}$ 值减去土壤孔隙水中的 $\delta^{13} \mathrm{CH}_{4}$ 值来估算 $\varepsilon_{\text {传榆 }}$ Conrad 和 Klose, 2005) 显然存在很大的 不确定性. 非水稻生长期, 稻田土壤产生的 $\mathrm{CH}_{4}$ 向大 气释放时, 大部分会被土水界面的甲烷氧化细菌氧 化, 剩余下来进入表层水中的 $\delta^{13} \mathrm{CH}_{4}$ 则能一定程度 上代表氧化后但还未经气泡或液相扩散传输的 $\delta^{13} \mathrm{CH}_{4}$. 甲烷氧化菌优先氧化 ${ }^{12} \mathrm{CH}_{4}$ 而使得未被氧化 的 $\mathrm{CH}_{4}$ 富集 ${ }^{13} \mathrm{C}$ (Whiticar, 1999; Venkiteswaran 和 Schiff,

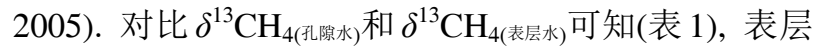
水中的 $\mathrm{CH}_{4}$ 的确发生了强烈的氧化作用. 因此, 可由
田间排放的 $\delta^{13} \mathrm{CH}_{4}$ 值减去土壤表层水中的 $\delta^{13} \mathrm{CH}_{4}$ 值

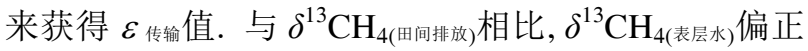
$3.0 \%$ 6.7\% o(表 1)，这表明土壤表层水中的 $\mathrm{CH}_{4}$ 在向 大气传输的过程中发生了 $3.0 \%$ 6.7\% 的碳同位素分

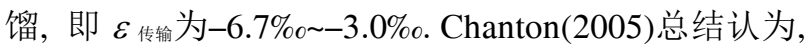
气泡和液相扩散使得通过水-气界面的 $\mathrm{CH}_{4}$ 几乎不发 生或发生很小的碳同位素分馏, 本试验结果表明, 稻 田土壤表层水中的 $\mathrm{CH}_{4}$ 通过气泡和液相扩散释放到 大气时发生了一定程度的碳同位素分馏. 有关稻田 $\mathrm{CH}_{4}$ 通过气泡或液相扩散传输而发生碳同位素分馏 的研究报道仍然还很少, 值得进一步深入研究.

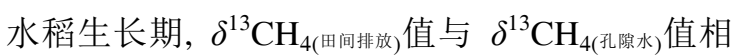

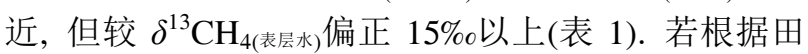
间排放的 $\delta^{13} \mathrm{CH}_{4}$ 值减去土壤表层水中的 $\delta^{13} \mathrm{CH}_{4}$ 值获 得 $\varepsilon$ 传船值, 那么它在-16.6\% - 15.2\%。. 水稻生长期, 稻田土壤产生的 $\mathrm{CH}_{4}$ 绝大部分通过植株通气组织传 输释放至大气中, 只有很少一部分 $\mathrm{CH}_{4}$ 通过气泡和 液相扩散途径排放, 且产生的 $\mathrm{CH}_{4}$ 大部分在根一土 界面被氧化(Schütz等, 1989; Sass 等, 1990; Frenzel 等,

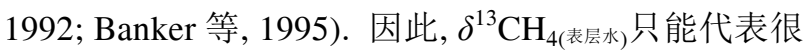
少一部分已被甲烷氧化细菌氧化但还未经气泡或液 相扩散传输的 $\delta^{13} \mathrm{CH}_{4}$, 而 $\delta^{13} \mathrm{CH}_{4 \text { (田间排放 }}$ 代表的是大部 分通过植株通气组织传输排放的 $\delta^{13} \mathrm{CH}_{4}$. 由此可见,

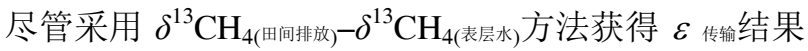
与以往研究报道相似(表 2), 但运用该方法来估算水稻 生长期的 $\varepsilon$ 传输本身并不是很科学, 不值得提倡和推广.

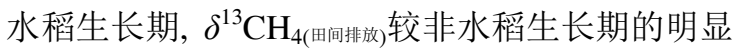

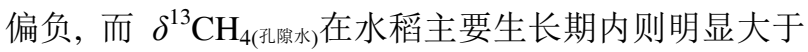

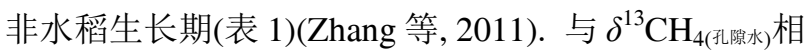

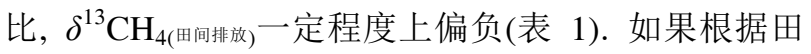

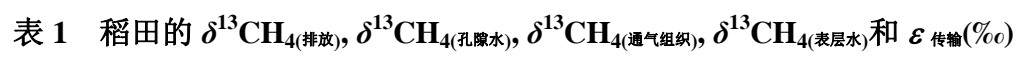

\begin{tabular}{|c|c|c|c|c|c|c|}
\hline 测定方法 & 采样日期 & $\delta^{13} \mathrm{CH}_{4 \text { (排放) }}$ & $\delta^{13} \mathrm{CH}_{4 \text { (孔㩐水) }}$ & $\delta^{13} \mathrm{CH}_{4 \text { (通气组织) }}$ & $\delta^{13} \mathrm{CH}_{4 \text { (表层水) }}$ & $\varepsilon$ 传输 \\
\hline \multirow{4}{*}{$\begin{array}{l}\varepsilon_{\text {传输 }=} \delta^{13} \mathrm{CH}_{4 \text { (田间排放 })}- \\
\delta^{13} \mathrm{CH}_{4 \text { (表层水) }}\end{array}$} & $2009-05-27$ & $-43.7 \pm 7.8$ & $-61.2 \pm 3.6$ & - & $-40.7 \pm 2.3$ & -3.0 \\
\hline & 2009-06-05 & $-52.2 \pm 4.9$ & $-66.0 \pm 5.8$ & - & $-45.5 \pm 0.4$ & -6.7 \\
\hline & 2009-07-12 & $-65.4 \pm 5.0$ & $-66.0 \pm 1.6$ & - & $-50.2 \pm 1.7$ & -15.2 \\
\hline & 2009-08-15 & $-59.7 \pm 6.4$ & $-56.5 \pm 1.0$ & - & $-43.1 \pm 1.3$ & -16.6 \\
\hline \multirow{4}{*}{$\begin{array}{l}\varepsilon_{\text {传输 }}=\delta^{13} \mathrm{CH}_{4(} \\
\delta^{13} \mathrm{CH}_{4 \text { (飞隙水 })}\end{array}$} & $2009-07-24$ & $-67.6 \pm 1.3$ & $-54.4 \pm 3.3$ & - & - & -13.2 \\
\hline & $2009-08-15$ & $-59.7 \pm 6.4$ & $-56.5 \pm 1.0$ & - & - & -3.1 \\
\hline & 2009-08-24 & $-64.0 \pm 3.0$ & $-56.5 \pm 6.7$ & - & - & -7.5 \\
\hline & 2009-09-05 & $-64.2 \pm 3.5$ & $-63.1 \pm 5.4$ & - & - & -1.1 \\
\hline \multirow{3}{*}{$\begin{array}{l}\varepsilon_{\text {传输 }=} \delta^{13} \mathrm{CH}_{4(\text { 植株排放 })}- \\
\delta^{13} \mathrm{CH}_{4 \text { (通气组织 })}\end{array}$} & $2009-08-02$ & $-60.9 \pm 4.1$ & - & $-48.5 \pm 3.2$ & - & -12.5 \\
\hline & 2009-08-27 & $-58.8 \pm 3.3$ & - & $-42.4 \pm 3.0$ & - & -16.3 \\
\hline & 2009-10-02 & $-59.5 \pm 2.8$ & - & $-48.6 \pm 1.0$ & - & -10.9 \\
\hline
\end{tabular}


表 2 稻田 $\mathrm{CH}_{4}$ 传输的碳同位素分馏系数 $\varepsilon_{\text {传输 }}(\% o)^{\mathrm{a})}$

\begin{tabular}{|c|c|c|c|c|}
\hline 测定方法 & 试验地点 & 水稻品种 & $\varepsilon_{\text {传输 }}$ & 参考文献 \\
\hline$\varepsilon_{\text {传输 }=} \delta^{13} \mathrm{CH}_{4 \text { (田间排放) }}-$ & 德国 & Rome & $-18 \sim-9.0$ & Conrad 和 Klose (2005) \\
\hline$\delta^{13} \mathrm{CH}_{4 \text { (飞隙水 })}$ & 中国 & Huajing 3 & $-13.2 \sim-1.1$ & 本研究 \\
\hline \multirow{6}{*}{$\begin{array}{l}\varepsilon_{\text {传输 }=} \delta^{13} \mathrm{CH}_{4(\text { 田间排放 })}- \\
\delta^{13} \mathrm{CH}_{4(\text { 通气组织 })}\end{array}$} & 美国 & Jasmine 85 & $-12.2 \pm 1.4$ & Tyler 等(1997) \\
\hline & 美国 & $\begin{array}{l}\text { Texmont } \\
\text { Lemont }\end{array}$ & $\begin{array}{l}-12.1 \\
-10.7\end{array}$ & Chanton 等(1997) \\
\hline & 美国 & Lemont 和 Mars & $-11.4 \pm 2.2$ & Bilek 等(1999) \\
\hline & 意大利 & Koral & $-16.0 \sim-9.8$ & Krüger 等(2002) \\
\hline & 意大利 & Koral & $-18.4 \pm 2.1$ & Krüger 和 Frenzel(2003) \\
\hline & 日本 & Koshihikari & $-16 \sim-11$ & Han 等(2005) \\
\hline$\varepsilon_{\text {传输 }=} \delta^{13} \mathrm{CH}_{4 \text { (蜢株排放) }}-$ & 中国 & Huajing 3 & $-16.3 \sim-10.9$ & 本研究 \\
\hline$\delta^{13} \mathrm{CH}_{4 \text { (通气组约) }}$ & 中国 & Huajing 3 & $-12.0 \sim-8.0$ & Zhang 等(2013) \\
\hline$\varepsilon_{\text {传输 }} \delta^{13} \mathrm{CH}_{4 \text { (田间排放) }}$ & \multirow[t]{2}{*}{ 中国 } & Huajing 3 & $-16.6 \sim-15.2$ & \multirow{2}{*}{ 本研究 } \\
\hline$-\delta^{13} \mathrm{CH}_{4 \text { (表层水) }}$ & & 无* & $-6.7 \sim-3.0$ & \\
\hline
\end{tabular}

间排放的 $\delta^{13} \mathrm{CH}_{4}$ 值减去土壤孔隙水中的 $\delta^{13} \mathrm{CH}_{4}$ 值获

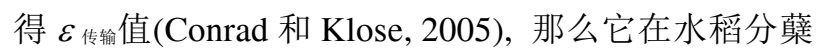
初期为 $-13.2 \%$, 拔节孕穗初期增大至 $-3.1 \%$, 后期则 减小到-7.5\%o, 灌浆期又增大至 $-1.1 \%$ o(表 1). 理论上, 分㮐初期水稻植株通气组织发育不完全, 其传输能 力相对较弱, 此时经通气组织传输释放的 $\mathrm{CH}_{4}$ 所发 生的碳同位素分馏可能较小, 由于同位素分馏系数

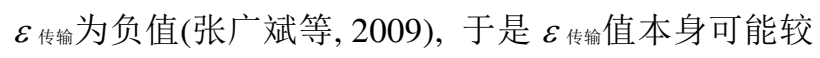
大, 而拔节孕穗期和灌浆期植株根系及其通气组织 发达, 传输能力强, 传输 $\mathrm{CH}_{4}$ 产生的碳同位素分馏可 能较大, 此时 $\varepsilon$ 传制值则相对较小. 意大利盆栽试验也 发现, 水稻分菜初期 $\mathrm{CH}_{4}$ 传输产生的碳同位素分馏 最小, 随后逐渐增大, 拔节孕穗期和灌浆期达到最大 值(Conrad 和 Klose, 2005). 本研究结果中, 分馏系数 $\varepsilon$ 传输值在分繤初期最小, 拔节孕穗期和灌浆期反而最 大, 与 Conrad 等(2005)观测结果的变化趋势刚好相

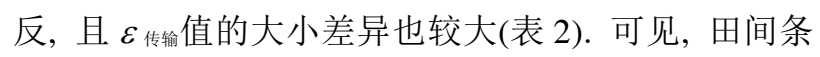

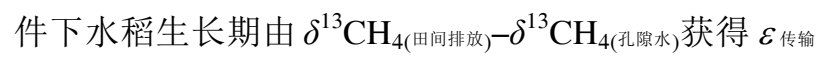

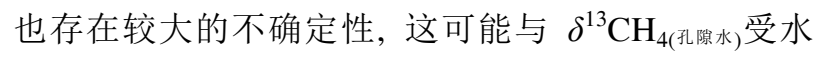
稻植株根系氧化的影响与温室盆栽的不同有关, 相 关原因有待进一步深入查明. 田间实际情况相当复 杂, 土壤孔隙水中的 $\mathrm{CH}_{4}$ 是否受到根际甲烷氧化菌

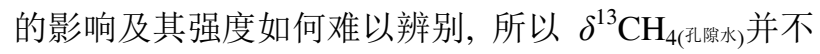
都能较好地代表已被甲烷氧化细菌氧化但还未经水稻

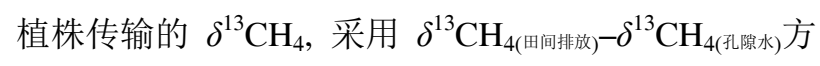
法来估算 $\varepsilon$ 传输可能要根据试验的实际情况而定.

水稻植株割苗前排放的 $\delta^{13} \mathrm{CH}_{4}\left(\delta^{13} \mathrm{CH}_{4 \text { (植侏排放 })}\right)$ 值

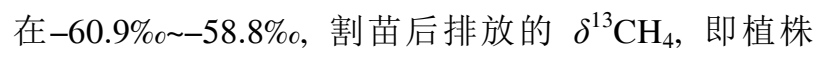

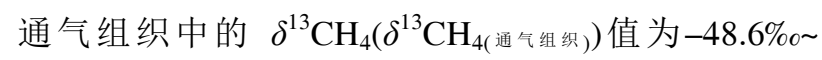

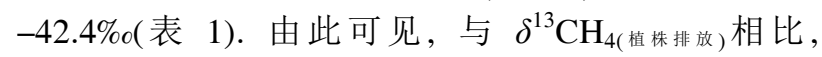

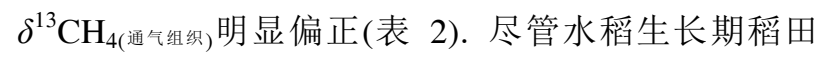
$\mathrm{CH}_{4}$ 绝大部分是通过植株通气组织传输释放, 但仍有 一小部分是通过气泡和液相扩散途径排放, 此时田 间排放的 $\delta^{13} \mathrm{CH}_{4}$ 并不能完全等同于水稻植株排放的 $\delta^{13} \mathrm{CH}_{4}$. 特别是在水稻生长初期, 水稻植株根系及通 气组织不发达, 气泡和液相扩散是稻田 $\mathrm{CH}_{4}$ 排放很 重要的途径, 根据田间排放的 $\delta^{13} \mathrm{CH}_{4}$ 值减去植株通 气组织中的 $\delta^{13} \mathrm{CH}_{4}$ 值获得 $\varepsilon_{\text {传款 }}$ (Tyler 等, 1997; Bilek 等, 1999; Krüger 等, 2002; Krüger 和 Frenzel, 2003)值 显然存在一定的不确定性. 采用分隔+切割观测方法, 能同时获得植株排放的 $\delta^{13} \mathrm{CH}_{4}$ 和植株通气组织中的

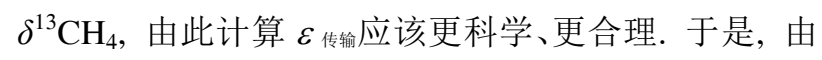

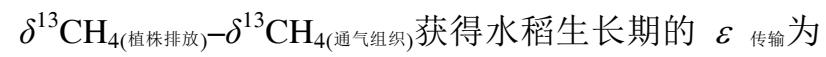
$-16.3 \%$ - 10.9\%o, 这与我们另外一个田间试验的结 果也相近 (表 2). 水稻生长初期, 随着植株根系及通 气组织逐渐发达, $\mathrm{CH}_{4}$ 传输能力也随之增强, 传输过 程中产生的碳同位素分馏可能也相应增大, 并在水

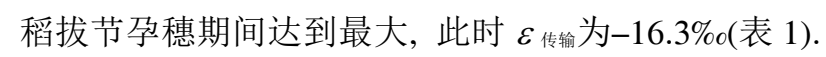
水稻生长后期, 根系及通气组织逐渐老化, 其传输 $\mathrm{CH}_{4}$ 的能力也可能随之减弱, 于是传输 $\mathrm{CH}_{4}$ 过程中发 生的碳同位素分馏也相应减小, $\varepsilon$ 传制为 $-10.9 \%$ (表 1 ), 这与以往研究结果的大小及其季节变化趋势相似 (Krüger 等, 2002; Conrad 和 Klose, 2005).

水稻生长期, 稻田 $\varepsilon$ 传制可能还受水稻品种及田间 水、肥管理等措施的影响 (表 2), 且随水稻生长状况 而发生变化(Krüger 等, 2002; Conrad 和 Klose, 2005). 
因此, 很有必要建立一套统一的、既科学又可靠的观 测方法, 以消除上述各种影响来获得准确可靠的研 究结果, 分隔+切割观测方法可弥补以往研究方法的 不足; 非水稻生长期, 稻田 $\varepsilon$ 传输不同于水稻生长期, 且具有自身特有的观测方法, 密闭箱+注射器观测方 法能很好地实现这一研究目的. 综上所述, 这两种方 法既科学又可靠, 且操作简单, 容易掌握, 具有一定 研究价值及推广价值.

\section{3 结论}

密闭箱+注射器法以及分隔+切割法分别能较好
地研究非水稻生长期和水稻生长期稻田 $\mathrm{CH}_{4}$ 传输的 碳同位素分馏. 非水稻生长期, 稻田土壤产生的 $\mathrm{CH}_{4}$ 向大气传输释放时发生的碳同位素分馏较小, 分馏 系数 $\varepsilon_{\text {传输 }}\left(-6.7 \% 0 \sim-3.0 \%\right.$ ) 由田间排放的 $\delta^{13} \mathrm{CH}_{4}$ 值减去 表层水中的 $\delta^{13} \mathrm{CH}_{4}$ 值获得; 水稻生长期, 稻田土壤产 生的 $\mathrm{CH}_{4}$ 向大气传输释放时发生的碳同位素分馏相 对较大, 分馏系数 $\varepsilon$ 传输 $(-16.3 \%$ $-10.9 \%$ ) 由植株排放 的 $\delta^{13} \mathrm{CH}_{4}$ 值减去通气组织中的 $\delta^{13} \mathrm{CH}_{4}$ 值获得. 这两 种方法获得的 $\varepsilon$ 传输科学、可靠, 且操作方法简单、易 掌握, 具有研究及推广价值. 关于水稻品种及田间 水、肥管理措施对 $\varepsilon$ 传输的影响尚需深入研究.

\section{参考文献}

曹亚澄, 孙国庆, 韩勇, 等. 2008. 大气浓度下 $\mathrm{N}_{2} \mathrm{O}, \mathrm{CH}_{4}$ 和 $\mathrm{CO}_{2}$ 中氮、碳和氧稳定同位素比值的质谱测定. 土壤学报, 45: 249-258

张广斌, 马静, 徐华, 等. 2009. 稳定性碳同位素方法在稻田甲烷研究中的应用. 土壤学报, 46: 676-684

Banker B C, Kludze H K, Alford D P, et al. 1995. Methane sources and sinks in paddy rice soils: Relationship to emissions. Agric Ecosyst Environ, 53: 243-251

Bilek R S, Tyler S C, Sass R L, et al. 1999. Differences in $\mathrm{CH}_{4}$ oxidation and pathways of production between rice cultivars deduced from measurements of $\mathrm{CH}_{4}$ flux and $\delta^{13} \mathrm{C}$ of $\mathrm{CH}_{4}$ and $\mathrm{CO}_{2}$. Glob Biogeochem Cycle, 13: 1029-1044

Chanton J P. 2005. The effect of gas transport on the isotope signature of methane in wetlands. Org Geochem, 36: 753-768

Chanton J P, Whiting G J, Blair N E, et al. 1997. Methane emission from rice: Stable isotope, diurnal variations, and $\mathrm{CO}_{2}$ exchange. Glob Biogeochem Cycle, 11: 15-27

Conrad R, Klose M. 2005. Effect of potassium phosphate fertilization on production and emission of methane and its ${ }^{13} \mathrm{C}$-stable isotope composition in rice microcosms. Soil Biol Biochem, 37: 2099-2108

Happell J D, Chanton J P, Showers W S. 1994. The influence of methane oxidation on the stable isotopic composition of methane emitted from Florida swamp forests. Geochim Cosmochim Acta, 58: 4377-4388

Happell J D, Chanton J P, Showers W S. 1995. Methane transfer across the water-air interface in stagnant wooded swamps of Florida. Limnol Oceanogr, 40: 290-298

Frenzel P, Rothfuss F, Conrad R. 1992. Oxygen profiles and methane turnover in a flooded rice microcosm. Biol Fert Soils, 14: 84-89

Han G H, Yoshikoshi H, Nagai H, et al. 2005. Late growing season $\mathrm{CH}_{4}$ budget in a rice paddy determined using stable carbon isotope, emission flux and soil storage measurements. Org Geochem, 36: 789-801

Knox M, Quay P D, Wilbur D. 1992. Kinetic isotope fractionation during air-water gas transfer of $\mathrm{O}_{2}, \mathrm{~N}_{2} \mathrm{CH}_{4}$ and $\mathrm{H}_{2}$. J Geophys Res, 97 : 20335-20343

Krüger M, Eller G, Conrad R, et al. 2002. Seasonal variation in pathways of $\mathrm{CH}_{4}$ production and in $\mathrm{CH}_{4}$ oxidation in rice fields determined by stable carbon isotopes and specific inhibitors. Glob Change Biol, 8: 265-280

Krüger M, Frenzel P. 2003. Effects of $\mathrm{N}$-fertilisation on $\mathrm{CH}_{4}$ oxidation and production, and consequences for $\mathrm{CH}_{4}$ emissions from microcosms and rice fields. Glob Change Biol, 9: 773-784

Sass R L, Fisher F M, Harcombe P A. 1990. Methane production and emission in a Texas rice field. Glob Biogeochem Cycle, 4: 47-68

Schütz H, Seiler W, Conrad R. 1989. Processes involved in formation and emission of methane in rice paddies. Biogeochemistry, 7: 33-53

Tyler S C, Bilek R S, Sass R L, et al. 1997. Methane oxidation and pathways of production in a Texas paddy field deduced from measurements of flux, $\delta^{13} \mathrm{C}$, and $\delta \mathrm{D}$ of $\mathrm{CH}_{4}$. Glob Biogeochem Cycle, 11: 323-348

Venkiteswaran J J, Schiff S L. 2005. Methane oxidation: Isotopic enrichment factors in freshwater boreal reservoirs. Appl Geochem, 20: 683-690

Whiticar M J. 1999. Carbon and hydrogen isotope systematics of bacterial formation and oxidation of methane. Chem Geol, 161: 291-314

Zhang G B, Ji Y, Ma J, et al. 2013. Pathway of $\mathrm{CH}_{4}$ production, fraction of $\mathrm{CH}_{4}$ oxidized, and ${ }^{13} \mathrm{C}$ isotope fractionation in a straw incorporated rice field. Biogeosciences, 10: 3375-3389

Zhang G B, Zhang X Y, Ji Y, et al. 2011. Carbon isotopic composition, methanogenic pathway and fraction of $\mathrm{CH}_{4}$ oxidized in rice field flooded all year round. J Geophys Res: 116: G04025 